\title{
Criminologie
}

\section{La libération conditionnelle : le point de vue des acteurs}

\section{Carmen Arsenault}

Volume 14, numéro 2, 1981

Libération conditionnelle : évolution et application (1899-1981)

URI : https://id.erudit.org/iderudit/017139ar

DOI : https://doi.org/10.7202/017139ar

Aller au sommaire du numéro

Éditeur(s)

Les Presses de l'Université de Montréal

ISSN

0316-0041 (imprimé)

1492-1367 (numérique)

Découvrir la revue

Citer cet article

Arsenault, C. (1981). La libération conditionnelle : le point de vue des acteurs. Criminologie, 14(2), 41-60. https://doi.org/10.7202/017139ar d'utilisation que vous pouvez consulter en ligne.

https://apropos.erudit.org/fr/usagers/politique-dutilisation/ 


\section{LA LIBÉRATION CONDITIONNELLE : \\ LE POINT DE VUE DES ACTEURS}

Carmen Arsenault

Au cours de l'été 1980 , le Bureau des libérations conditionnelles de l'ouest de Montréal et l'École de criminologie de l'Université de Montréal entreprenaient conjointement la première étape d'une étude portant sur la libération conditionnelle.

Dans cette première phase exploratoire, l'étude visait la connaissance de la réalité vécue quotidiennement par les agents des libérations conditionnelles afin de tracer un état de la situation de la pratique actuelle. Cependant, un second objectif (initialement réservé à une étape ultérieure de la recherche) s'est rapidement greffé au précédent; en effet, il nous est apparu de plus en plus évident qu'une étude sur la libération conditionnelle ne pouvait faire entièrement abstraction de la réalité vécue par les libérés eux-mêmes en raison de l'étroite interdépendance entre les agents et leurs clients.

La réalisation de cette étude exploratoire centrée sur le vécu imposait l'emploi d'une méthodologie qualitative. Nous avons privilégié l'entretien à tendance non directive comme instrument de cueillette des données afin d'obtenir une vision en profondeur de l'expérience personnelle de chaque répondant. Les entrevues étaient structurées autour de certains thèmes déjà prévus lors de l'élaboration du projet d'étude, quoique en cours d'entretiens les répondants abordaient généralement d'eux-mêmes la plupart de ces thèmes en fonction de leur position d'agents ou de libérés : images réciproques, nature des relations et incidences, problèmes du libéré, attentes respectives, aspects aide et contrôle, relations avec la police, etc. Les agents abordèrent aussi les satisfactions, les difficultés et les déceptions provenant de leur travail, etc.

En raison des exigences de notre méthodologie qualitative, nous avons dû limiter notre échantillon à huit agents des libérations conditionnelles et deux ex-détenus (aux informations reçues de ces deux derniers répondants s'ajoutent quelques données obtenues fortuitement auprès de quatre autres libérés conditionnels). Les agents (cinq femmes et trois hommes) sont affectés à l'un ou à l'autre des six bureaux des libérations condi- 
tionnelles de la région de Montréal. Sauf en ce qui concerne un agent, ils sont âgés d'environ 30 ans et ont deux, quatre, cinq ou six années d'expérience comme agent des libérations conditionnelles. Ces agents sont, pour la plupart, criminologues mais d'autres sont travailleur social, diplômé en sciences sociales ou autre. Ils ont aimablement accordé des entrevues d'une durée moyenne de deux heures. Des deux répondants ex-détenus, l'un avait terminé sa surveillance obligatoire alors que l'autre était encore en libération conditionnelle. Ils avaient été incarcérés respectivement 4 ans et 15 ans, principalement dans un pénitencier à sécurité maximale. Nous sommes conscients que ces deux répondants forment un groupe trop restreint pour apporter des informations représentatives de la surveillance telle que prévue par la majorité des ex-détenus. Toutefois, à cette étape-ci de notre recherche, ces informations nous sont très utiles en tant que premier aperçu du vécu en période de surveillance.

Ainsi, en dépit des réserves que notre échantillon peut susciter, nous exposerons néanmoins dans cet article les trois dimensions qui se sont révélées particulièrement pertinentes à la lumière des données recueillies. Premièrement, il ressort que, selon la position occupée par les personnes impliquées dans le système des libérations conditionnelles, les perceptions énoncées quant à la représentation du rôle de l'agent des libérations conditionnelles sont fort différentes. Deuxièmement, nous énumérerons les différents outils utilisés par l'agent qui doit, d'une part, maintenir un contrôle et, d'autre part, assurer une relation d'aide aux libérés tout en mentionnant en contrepartie les réactions de ces derniers à ces mesures. Enfin, nous toucherons le dilemme aide-contrôle et nous verrons comment, dans sa pratique quotidienne, l'agent compose à l'intérieur de ce double aspect de sa fonction.

PERCEPTIONS DIFFÉRENTES DU RÔLE DE L'AGENT

D'après les personnes interviewées, le rôle de l'agent a une signification fort différente selon qui, du gestionnaire, de l'agent ou du libéré, définit et interprète la fonction de cet intervenant.

C'est à travers le discours des agents que fut décrite la place hégémonique accordée au contrôle par les gestionnaires du Service correctionel canadien. Tous les agents constatent que depuis quelques années, le Service donne de plus en plus la primauté au contrôle : contrôle des libérés et contrôle des agents eux-mêmes. 
Perception du Service correctionnel canadien : contrôle du libéré

Les agents mentionnent d'abord que le Service s'est graduellement éloigné de l'idéal de la réhabilitation poursuivi au début des années '70; ces derniers notent à regret que le rôle de l'agent s'est ainsi modifié sensiblement au cours des ans. Peu importe leur expérience dans la fonction, il apparaît aux agents que le Service exige d'eux qu'ils soient d'abord des agents de contrôle puisque les nouvelles directives accentuent et définissent clairement cet aspect de leur rôle auprès des libérés reléguant au second plan l'aspect assistance à la clientèle.

Axées sur le contrôle et non sur la création de meilleurs instruments de réinsertion sociale, les normes de surveillance ne sont pas fonction des intérêts des libérés et sont fort éloignées de la réalité vécue par les agents et leurs clients. La sévérité et la fixité de ces normes contribuent à brimer à la fois les libérés et les agents.

Les principales normes de surveillance qui affectent le plus les agents dans leur travail auprès de la clientèle sont : le même rythme de surveillance imposée à tous les clients, la directive relative aux visites dans le milieu, le devoir de rapporter à la Commission nationale des libérations conditionnelles les délits mineurs des libérés et la rédaction de rapports trimestriels. Ainsi, le même rythme de surveillance imposé à tous les clients, qu'ils aient ou non besoin de ces rencontres avec l'agent, constituent une perte de temps pour les agents en plus de les empêcher de s'occuper plus activement de ceux qui bénéficieraient d'une surveillance intensive. La directive relative aux visites dans le milieu impose à l'agent de rencontrer le libéré au bureau et dans son milieu, dans un rapport de 50-50; cette norme, défendable théoriquement, peut être désagréable dans son exécution. Du point de vue de l'agent dans certains cas, ces visites sont jugées impertinentes en raison de leur inutilité ou de la position confuse dans laquelle peut parfois se trouver l'agent (ex. : alcooliques, personnes de sexe opposé, etc.). Quant aux libérés, il arrive que certains d'entre eux ou que leur entourage ne souhaitent pas la venue de l'agent. Nous parlerons ultérieurement des autres éléments mentionnés ci-haut.

Perception du Service correctionnel canadien : contrôle de l'agent

Les agents constatent un alourdissement considérable de leur tâche au niveau administratif. Les exigences du Service sont telles que les agents consacrent la moitié de leur temps en travail bureau- 
cratique. Quoique les agents comprennent très bien la nécessité d'un aspect administratif afin d'assurer un suivi de l'évolution des cas, ils s'insurgent toutefois contre l'importance exagérée que le Service attribue à cet aspect. Par exemple, ils conçoivent mal l'utilité de la rédaction obligatoire de rapports trimestriels pour chaque libéré; dans leur pratique quotidienne, cette directive est jugée impertinente et même vaine. Tous les trois mois, les agents cherchent quoi écrire sur le client de qui il n'y a rien à dire puisqu'il ne s'est produit aucun changement dans sa vie : il a toujours la même adresse, le même emploi, la même femme et il semble toujours bien se porter.

L'importance de l'aspect administratif de la fonction de l'agent combinée à des normes de surveillance imposées qui régissent le travail avec le client amènent les agents à percevoir le travail tel qu'il est défini par le système comme un travail presque essentiellement bureaucratique. Alors que l'agent accorde la priorité à son travail auprès du client, il a l'impression que le Service, par ses exigences, repousse au second plan ce travail auprès du client. Ainsi, l'agent perd la latitude nécessaire pour mener des interventions adaptées aux besoins spécifiques de chaque libéré et il souffre d'être lui-même soumis à un sérieux contrôle par le biais des tâches administratives qu'il accomplit et qui sont sujettes à vérification par ses supérieurs. Bref, dans l'ensemble de leur fonction, les agents ont perdu leur sensation de liberté et s'indignent du peu de considération que les autorités accordent à leur statut professionnel.

Perception des agents

Quoique l'agent est conscient que les politiques du Service s'orientent toujours davantage vers la prédominance du contrôle, il demeure que son intérêt est avant tout centré vers la relation d'aide qu'il peut établir avec le libéré. Selon les agents, cet aspect de la surveillance est sans contredit l'élément le plus important et le plus valable de leur fonction parce qu'il leur semble que ce n'est pas par le contrôle mais plutôt par la relation d'aide qu'on peut espérer modifier le comportement social d'un individu. L'un des agents s'exprime ainsi :

Le rôle que je vais prendre moi, à travers çà, c'est d'en arriver à ce que l'individu soit plus réaliste, plus conscient et puisse partir de son expérience de vie pour se projeter et en arriver à une meilleure réalisation de soi-même et à une meilleure compréhension de soi-même [...] qu'on soit ca- 
pable d'établir un contact qui lui permette de mieux réaliser ce que c'est sa vie en société.

N'oubliant pas qu'au moment de leur engagement dans le Service, les agents ont accepté le mandat de superviser des personnes encore soumises aux impératifs d'une sentence légale, ils conçoivent et acceptent la dimension contrôle de leur rôle comme faisant partie des règles du jeu. Cependant, vu le peu d'impact qu'ils reconnaissent au contrôle du libéré, l'actuelle philosophie du Service et le rôle qui leur est imposé révèlent, selon eux, l'intention première des gestionnaires d'entretenir dans le public l'image illusoire d'une protection sociale adéquate.

\section{Perception du libéré}

D'après l'expérience des libérés, ceux-ci perçoivent la surveillance comme une mesure de contrôle uniquement et ils s'accommodent assez bien des modalités de contrôle inscrites sur leur certificat de libération; cela, surtout si le sort leur désigne un agent «correct », c'est-à-dire un agent qui, par ses questions, ne s'immisce pas trop profondément dans leur intimité et qui n'exerce pas son autorité d'une façon qu'ils jugent trop arbritraire. Par ailleurs, la forte conviction des pouvoirs répressifs attribués à l'agent conditionne leur image de ce dernier qu'ils perçoivent avant tout comme une personne à craindre ce qui les amène également à redouter, à refuser ou à réfuter toute prétention à une relation d'aide en contexte d'autorité formelle. D'autres libérés ignoreront tout simplement l'existence d'une relation d'aide, tel ce répondant :

Je ne me suis pas senti ni surveillé ni aidé [...]. Dans mon cas, je ne vois pas ce qui, en quoi ce qu'il peut m'avoir aidé; disons que je m'en suis pas rendu compte en tout cas.

Les prochains paragraphes exposeront davantage le vécu du libéré relativement à la surveillance exercée par son agent.

LES INTERVENTIONS DE L'AGENT AUPRÈS DES LIBÉRÉS

Les interventions de l'agent des libérations conditionnelles visent à empêcher la récidive et à favoriser la réinsertion sociale des libérés; ces objectifs s'inscrivent dans une perspective plus vaste soit la protection de la société. Une des dimensions essentielles de notre étude porte donc sur la matérialisation de la surveillance des libérés dans ses aspects contrôle et aide. Nous exposerons ici, parallèlement, les interventions des agents qui nous semblent les plus significatives et les perceptions et réponses des libérés à celles-ci. 


\section{Relation}

D'abord, il nous faut préciser que les agents reconnaissent que la nature de la relation qui existe entre eux et leurs clients influe sur la qualité de la surveillance. Établir un bon contact avec les clients devient l'aspect prioritaire de leur fonction. Conscients de l'image stéréotypée des libérés à leur endroit et de la méfiance «normale » que suscite leur identification au système carcéral, les agents considèrent qu'il leur revient de créer un climat de confiance mais cela exige un grand investissement de leur part. En ce contexte d'autorité, vaincre graduellement la méfiance est la plus grande difficulté rencontrée dans leur travail. Ils parviendraient à dissiper les angoisses en agissant avec beaucoup de patience et de diplomatie c'est-à-dire en évitant les intromissions trop hâtives ou trop brusques dans la vie intime des libérés. C'est ainsi qu'en dépit de l'intransigeance des conditions du certificat de libération avec lesquelles agents et libérés ont à composer, les agents font davantage valoir aux libérés leur intention première qui est de leur fournir aide et support pendant la période de surveillance.

Mais l'approche est peut-être d'abord de lui expliquer d'une façon très précise ce qu'il doit s'attendre en libération conditionnelle, les conditions qu'il a à observer. Et puis le caractère d'entregent entre l'agent et lui : essayer peut-être à briser dès le départ le mythe qu'on va le surveiller pour essayer de le prendre en défaut; çà lui est dit çà au pénitencier par d'autres qui ont récidivé par exemple. Ils n'ont pas des mots tendres à l'égard des libérations conditionnelles puis çà se propage. A toutes fins pratiques, il est presque induit en erreur sur notre fonction face à lui, à son égard. Si on peut le rassurer là-dessus pour lui faire comprendre que s'il a été libéré, c'est certainement pas pour essayer de le prendre en défaut et le rentrer aussitôt. Qu'on est, un petit peu comme l'annonce de Steinberg, on est sur son côté, sur son bord.

Selon les agents, ces sentiments de méfiance persistent rarement jusqu'à la fin de la période de surveillance; toutefois, ils conviennent qu'il est rare aussi d'obtenir la confiance totale des clients. Mais, de façon générale, les agents affirment avoir des relations bonnes ou satisfaisantes avec la plupart de leurs clients. La relation est jugée satisfaisante lorsque le libéré démontre une attitude ouverte lors de ses rendez-vous, qu'il parle volontiers de ses activités (travail, loisirs, famille, etc.) et de ses ambitions, qu'il accepte de discuter avec son agent, bref, lorsqu'il ne donne pas l'impression qu'il ne vient que par formalité. En ce sens, la 
relation avec le client dit «criminel professionnel » (sur qui la surveillance est perçue comme étant inutile car elle n'a aucun impact négatif ou positif) apparaît satisfaisante et même intéressante puisque habituellement, il n'affiche pas une attitude rebelle et qu'il a la conversation facile malgré qu'on doute de jamais connaître le fond de sa pensée. La relation est également agréable avec le «criminel occasionnel» (défini comme le client idéal) qui s'ouvre facilement et demande volontiers l'avis de son agent après avoir déjà bien solutionné lui-même ses problèmes.

Pour leur part, tous les libérés rencontrés de manière formelle ou informelle entretiennent plus ou moins volontairement des sentiments de méfiance envers leur agent. Tel un leitmotiv, les pouvoirs répressifs attribués à l'agent reviennent sans cesse dans leurs propos. Voici quelle est la perception de l'agent et de son rôle par un de nos répondants-libérés qui définit sa relation comme « uniquement une relation de tolérance »:

Je respecte l'être humain qu'il y a là mais, en même temps, je suis conscient de sa fonction sociale et de son rôle. Et puis y'a rien que moi qui peux en souffrir au bout de la ligne si je perds çà de vue [...]. Je joue un rôle, je joue le rôle du prisonnier qui va se rapporter. Puis mon rôle c'est d'en dire le moins possible [. . . J. Je ne le regrette pas comme individu; c'est son rôle qui fait que la clôture qui est entre nous deux, je n'ai pas le droit de l'ignorer [...] parce que, selon ses valeurs, selon ses perceptions qu'il va avoir de mes mots, c'est une personne dangereuse pour moi [...]. Moi, je veux rien qu'il se contente de puncher quand c'est le temps de puncher.

Par contre, un agent et un autre libéré avaient établi «un excellent contact» (selon cet agent). Ce libéré dit s'être "quasiment fait ami "avec son agent; néanmoins, il ne parvint jamais à s'ouvrir à lui :

Quand même, je ne voulais pas dépasser une certaine étape parce qu'il n'était pas de mon milieu puis j'étais pas du sien puis y'avait toujours les règlements en-dessous [...]. Ce qui me bloquait, ce qui n'a pas aidé la chose, c'est que c'est un gars des libérations puis moi j'étais un détenu. Cà fais que disons, lui était sur le côté du gars qui arrête le mauvais puis moi j'ai toujours été mauvais, çà fait que c'est pour çà [...]. Mais après çà, on a jasé, il parlait de sa femme, je parlais de la mienne [...] c'était surtout, en fait, de sports. A part çà, je ne sais pas, je ne débloquais pas. $\mathbf{J}$ 'ai pas de misère d'habitude à me faire des amis mais [...]. En tout cas, moi, j'ai passé 18 mois avec lui et pourtant, j'ai essayé de lui faire 
confiance; j'essayais mais j'étais pas capable de lui faire confiance.

\section{Informations}

Connaissant l'attitude suspicieuse des libérés à l'égard de leur agent, nous tenterons de voir ci-après comment, concrètement, l'agent exerce sa surveillance et comment les libérés vivent cette situation.

Pour remplir l'un ou l'autre des deux aspects de sa fonction (contrôle et relation d'aide), l'agent doit disposer d'informations sur la situation réelle de chacun de ses clients. En période de surveillance, ces informations proviennent du libéré lui-même, des personnes de son milieu (famille, employeur ou autres) et de la police.

Nous savons déjà que les propos émis par les libérés devant leur agent sont mesurés avec prudence et ne donnent qu'une vision superficielle de leur situation. Et les données de notre étude laissent croire que les informations recueillies ailleurs par les agents sont aussi peu nombreuses; de plus, leur valeur semble bien relative tant en fonction du contrôle que de l'aide à assurer. C'est ainsi que les agents peuvent rarement se permettre de chercher des renseignements auprès d'employeurs qui, le plus souvent, ignorent le statut particulier de certains employés. Relativement à la police, c'est à l'unanimité que les agents ont souligné les relations tendues entre eux et les policiers et ont déploré leur absence de collaboration. Une attitude compréhensive et une meilleure coopération de la part des corps policiers faciliteraient grandement le travail de l'agent tant au niveau de l'aide que du contrôle des libérés. Ces derniers ne seraient plus victimes de harcèlements et, en cas de ré-arrestation pour délits mineurs par exemple, l'agent rapidement prévenu pourrait agir promptement et empêcher parfois une réincarcération évitable (lorsque cela fut possible, cette dernière intervention a favorisé beaucoup l'établissement ou l'augmentation de la confiance de certains libérés envers leur agent). Au niveau du contrôle de quelques clients au sujet desquels l'agent entretient des doutes, la police pourrait confirmer ces soupçons et/ou les prendre en filature.

Justement, du côté des répondants-libérés, l'idée de ce pouvoir qu'a l'agent «de mettre la police après eux » ne les quitte pas. En réalité, ils sont satisfaits du comportement des policiers envers eux et apprécient surtout le fait que le rapport à la police se limite 
à une simple formalité (confirmation d'adresse, de lieu de travail et de marque d'automobile, etc.). Et un libéré ajoute ceci :

La police elle, elle est très correcte; elle est moins achalante que l'agent des libérations conditionnelles parce qu'elle ne cherche pas à savoir tout ce que tu fais dans la vie.

Ainsi, disposant de peu d'informations sur ses clients, pour mieux les connaître, l'agent privilégie les visites dans les foyers, particulièrement ceux où il peut rencontrer les membres de la famille du libéré (mentionnons toutefois que, dans l'esprit des agents, ces visites au domicile visent autant le support que le contrôle des clients). Selon les agents, il leur est assez facile d'établir une bonne relation avec la femme ou les parents des clients qui, après quelque temps, en viennent à les seconder dans leur rôle de surveillant. Voici, telles que mentionnées dans les entrevues, les informations recueillies par l'agent lors de ces visites :

- situation familiale ou conjugale : bonne ou conflictuelle

- tempérament du libéré : agressif ou non, dominateur ou non, responsable ou non du budget familial

- situation financière

- détails sur la situation de travail

- motifs d'un congédiement

- heures du lever

- activités familiales

- achat de nouveaux meubles

- culture d'un plant de marijuana

- changement d'adresse non déclaré

- désir d'avoir un enfant

- nervosité non habituelle de la conjointe (interprétée parfois comme un signe de récidive)

- arrestation récente et détention à Parthenais

Aucun des agents ne reçut de personnes du milieu une quelconque dénonciation par rapport à des délits commis ou en voie de se commettre par les libérés. Il arrive que les agents reçoivent une information du genre «çà va mal » mais cette information est généralement relative à une situation familiale ou conjugale problématique.

Un de nos répondants-libérés vivait avec une femme pendant sa surveillance; jamais l'agent ne soupçonna (vérification faite) que cette femme le craignait au point de s'absenter pendant ses visites :

Ah! elle était rarement ici parce qu'elle se trouvait à travailler le soir [...] puis soit qu'elle sortait ou quelque chose de 
même. Elle aimait pas gros çà, elle avait peur de çà. Elle le prend pour un dangereux, elle dit : il va t'embarquer, c'est çi puis c'est çà.

Et voici quelle était la perception de ce même libéré face aux visites de son agent :

En tout cas, je me demande ben pourquoi il venait ici dans la maison. Il venait-tu pour sentir ou ben s'il venait pour se reposer? Je me demande ben pourquoi [...].

Modalités de contrôle

Les libérés et les agents perçoivent les conditions inscrites sur le certificat de libération et toutes les interventions de l'agent qui leur sont relatives comme des modalités de contrôle puisque tout manquement connu à l'une ou à l'autre de ces conditions expose en principe le contrevenant à un retour au pénitencier.

L'ensemble des entrevues réalisées avec les agents démontrent que ceux-ci sont en mesure de contrôler les libérés par trois moyens : la vérification des rapports à la police, la présence aux rendez-vous prévus par l'agent et l'examen de la situation de travail. Nous ne nous étendrons pas sur les deux premiers points mais il nous faut parler quelque peu du troisième. Le secteur du travail est celui auquel les agents accordent une importance primordiale puisqu'ils évaluent généralement le bon comportement social de leurs clients à partir de l'occupation d'un emploi et de la stabilité au travail.

En ce domaine, la force des pressions, le genre d'investigations ou le degré de tolérance des agents varient selon l'agent, la spécificité de sa clientèle et la prise en considération du contexte socio-économique. Ainsi, certains agents ne tolèrent pas le nonemploi de leurs clients qu'ils obligent à trouver un travail dans les plus brefs délais alors que d'autres sont plus patients, étant d'avis que l'obtention d'un emploi convenable et apprécié offre de meilleures garanties de réinsertion sociale. Certains agents exigent copie de tous les talons de chèques de paye, méthode que d'autres agents condamnent. Dans certains quartiers très défavorisés, l'agent est forcé d'accepter une situation de non-emploi pratiquement généralisée et jugée presque irrémédiable chez une clientèle définie comme hypothéquée sur tous les plans. D'autre part, les agents ne peuvent négliger l'impact de la situation socio-économique actuelle et du chômage qui sévit. Des agents, jadis sévères envers ceux qui démontraient une paresse face au travail, ont dû s'adapter à cette nouvelle réalité sociale tout en soulignant que 
leur rôle de surveillant en est complexifié. Se remémorant avec regret une époque plus favorable (préparation de l'Exposition internationale de Montréal en 1967 ou des Jeux olympiques de 1976 par exemple), un agent déclare : "Tous mes gars travaillaient; c'était facile la surveillance dans ce temps-là ».

Les libérés sont très conscients du contrôle exercé sur eux par le travail. L'un d'eux affirme : "Je leur dis ce qu'ils veulent entendre : je travaille ». En répondant à cette exigence, les libérés s'évitent tracasseries et mesures jugées arbitraires tel le resserrement du rythme de surveillance auquel fut soumis l'un d'eux (après deux ans de stabilité à un même emploi) pendant le mois de repos qu'il s'accorde avant un changement de travail.

Les agents ne peuvent s'assurer du respect intégral de plusieurs clauses du certificat de libération et, en effet, de nombreux bris de conditions échappent à leur connaissance particulièrement en ce qui concerne les dettes et les voyages non autorisés ainsi que le non-rapport d'infractions ou d'accidents à la circulation.

La clause obligeant les libérés à demander des autorisations de voyages apparaît comme étant la plus problématique. Aussi ne les demandent-ils pas toujours (et certains ne le font que très rarement) pour plusieurs raisons :

1) cette condition est jugée «enfantine et niaiseuse 》;

2) l'agent est susceptible de poser des questions et d'enquêter sur les personnes à rencontrer au cours du voyage;

3) sauf pour quelques événements spéciaux, personne dans la société n'est en mesure de toujours prévoir à l'avance ses déplacements;

4) cette exigence est sous-tendue par les soupçons qu'on entretient sur eux.

Et voici à ce sujet l'opinion d'un libéré :

[... ] c'est peut-être la seule finalement que je trouve, encore là, enfantine puis niaiseuse parce qu'on suppose que c'est absolument important d'avertir la police partout où tu vas être. Mais pourquoi? Ça veut dire donc, pour eux autres, que t'est toujours susceptible de commettre un crime. S'il y a un crime dans telle région, si tu étais là dans ce temps-là, donc c'est possible que ce soit toi. La confiance règne pas [...].

D'autre part, les libérés voient les préjudices qu'ils pourraient subir s'ils rapportaient leurs infractions au Code de la route (dans le cas d'un libéré de notre étude, l'accident dans 
lequel il fut impliqué), inscription dans leur dossier et utilisation éventuelle comme arguments de preuve d'un mauvais comportement social.

Cependant, les libérés comprennent très bien le rationnel de la clause traitant des armes puisqu'elle s'applique à tous les citoyens. En revanche, notons que l'obligation de demander la permission avant de se marier est très mal vue; selon eux, la question de leur mariage ne concerne pas l'agent des libérations conditionnelles.

Bref, les deux répondants-libérés de cette étude ne se sont sentis ni harcelés ni particulièrement surveillés. Voici ce que dit l'un d'eux dont la période de surveillance est terminée :

Moi, je ne me suis pas senti surveillé, je me sens comme je me sens là tu sais. Ça passait de même, je ne m'en rendais pas compte que j'étais sous libération conditionnelle.

Enfin, les agents reconnaissent leur inaptitude à contrôler parfaitement leurs clients. La structure de contrôle propre au Service des libérations conditionnelles n'est pas de l'ordre d'une surveillance policière. Au contraire, une surveillance de type policier s'inscrirait fort mal dans une perspective de réinsertion sociale en bloquant toute ouverture propice à une relation d'aide. C'est pourquoi les agents ne déplorent pas leur propre impuissance à contrôler eux-mêmes les libérés quoiqu'ils regrettent en même temps l'absence de collaboration entre leur agence et les agences policières.

\section{Relation d'aide}

Examinons maintenant ce qu'il en est de la relation d'aide dans le contexte de la libération conditionnelle. Précisons d'abord que chez les agents rencontrés, malgré leur désir évident d'aider, il est difficile de connaître la nature et les résultats de leurs interventions en ce sens. En abordant cette question, nous entrons dans un domaine d'incertitudes. Les agents ne donnent généralement qu'un aperçu de leur démarche globale tout en illustrant leurs propos par des exemples d'aide apportée dans quelques situations spécifiques, un peu inhabituelles et sporadiques. D'après ce qui ressort des entrevues, par aide, il faut entendre toutes les interventions de l'agent en vue de répondre ou de tenter de répondre aux besoins matériels et/ou psychologiques du client (notons que la plupart des agents ont substitué l'expression « réinsertion sociale » au mot « réhabilitation » puisque ce dernier 
mot réfère à quelque chose dont ils nient l'existence dans le système carcéral).

En ce qui concerne la relation d'aide, comme le souligne un agent, «il n'y a pas de recette » pouvant guider les agents dans leurs interventions; celles-ci reposent sur le jugement et les aptitudes personnelles de l'agent et exigent aussi la coopération du client. La relation d'aide est ainsi soumise à bien des impondérables : le refus d'aide de la part de certains clients, l'absence de besoins en ce sens chez bon nombre d'autres, l'inaptitude de l'agent à solutionner certains problèmes et le manque de ressources sociales.

De l'avis des agents, il y a effectivement des libérés qui refusent à leur agent un droit de regard sur leur vie personnelle et ne leur permettent aucune intervention allant au-delà de la 《surveillance technique ». Les agents ne s'acharnent généralement pas auprès de ces clients pour les motifs suivants : respect de l'individu, constat d'impuissance, non-croyance dans le succès d'une intervention imposée ou, vu le temps limité passé auprès de la clientèle, désir d'éviter un "gaspillage d'énergie ». Et les agents sont tous d'accords pour admettre qu'une partie de leur clientèle n'a nullement besoin de leur aide. Les propos suivants illustrent bien cette opinion générale tout en nous permettant d'identifier quand un agent juge qu'un libéré a ou n'a pas besoin d'aide :

Si tu as un libéré conditionnel qui est un individu qui a une très bonne organisation personnelle, je veux dire : l'individu travaille, il a un milieu familial stable. C'est comme tout le monde; je veux dire : c'est bas mais en général stable. Bien, c'est sûr que pour celui-là, tu vas pas chercher les bébites noires où il n'y en a pas. Parce qu'il y a effectivement des individus qui sont en libération conditionnelle qui n'ont pas besoin de nous; ils n'ont pas besoin d'être surveillés, qui viennent ici parce que c'est vraiment une loi, c'est tout et puis nous autres aussi on le sait.

Les agents ne perçoivent pas de différence majeure entre leurs clients (autant ceux libérés sous le régime de la surveillance obligatoire que ceux en libération conditionnelle) et les citoyens ordinaires tant au niveau de leurs difficultés que de leurs aspirations. Selon eux, l'expérience de l'emprisonnement est la seule caractéristique des ex-détenus et cette expérience spécifique exige une réadaptation à la vie en société. Face à cette réadaptation, l'agent intervient de deux façons : par une attitude compréhensive qui autorise un défoulement visant à éliminer la révolte entretenue 
contre le système et par les références fournies en vue de satisfaire certains besoins concrets : lieu d'hébergement (cas exceptionnels), recours temporaire aux allocations du Bien-être social et conseils quant à la recherche d'emploi. Mais d'après l'expérience des agents et des ex-détenus, il demeure que la réadaptation se fait graduellement et surtout par l'immersion dans une nouvelle réalité quotidienne.

S'inscrivant dans une relation d'aide, les autres interventions des agents touchent les secteurs suivants : le travail, les problèmes d'alcoolisme, les difficultés conjugales ou familiales ainsi que des questions diverses concernant l'amélioration de la personnalité psycho-sociale des clients.

Il semble qu'il n'est possible aux agents d'établir une relation d'aide qu'avec certains types de clients, principalement ceux qu'ils appellent les «motivés au départ », ceux qu'ils arrivent à motiver, les "dépendants » et ceux qu'ils ont obligés à réagir à la suite d'une provocation quelconque (suspension "thérapeutique», maintien de la surveillance du client par l'agent même qu'on pensait fuir en transférant dans un autre secteur, situation créant un état de défensive obligeant le client à dire au moins s'il est d'accord ou non avec les propos de l'agent, etc.). Ajoutons que, considérant le temps qui s'écoule avant qu'existent une bonne relation et un climat de confiance entre l'agent et le client, un de nos répondants fit part de la constatation suivante :

Mais pour en arriver à être capable de nous demander conseil, il faut qu'ils aient un bon bout de chemin de fait. Ça fait que c'est déjà la personne qui a le moins besoin de ton aide.

Selon les agents, c'est dans le secteur du travail que les libérés rencontrent leurs principaux et leurs plus considérables problèmes que l'actuelle conjoncture économique amplifie encore davantage. Le "délinquant ordinaire » (majorité de la clientèle), déjà handicapé par son dossier judiciaire, son absence plus ou moins prolongée du marché du travail, son inexpérience, son manque de formation et de relations, ne sait pas de plus comment chercher un emploi, ni comment se présenter devant un employeur éventuel.

L'agent réfère généralement ses clients au personnel du Centre de main-d'œuvre spécialement habilité à aider les exdétenus dans leur recherche d'emploi. Certains clients seront aussi dirigés vers des cours de formation. Directement auprès de leur 
clientèle, les agents poursuivent l'objectif suivant : stabiliser les libérés au travail. Cet objectif serait difficile à atteindre car l'exdétenu n'a pas l'habitude du travail (le pénitencier n'aide pas à développer de réelles habitudes de travail) et parce que, bien souvent, les emplois qui l'intéressent lui sont inaccessibles. Ainsi, l'agent, tel un orienteur non professionnel, doit aider le client dans l'évaluation de centres d'intérêts en accord avec sa réalité. De plus, comme les types de métiers accessibles aux libérés (ouvriers, journaliers, mécaniciens, etc.) ne permettent généralement pas d'aspirer à un niveau de vie élevé, les agents ont un immense travail à faire autour de l'acceptation de cette situation qui ne faciliterait pas la réinsertion sociale.

Dans le secteur du travail, les agents reconnaissent que leur apport est fort limité et ils déplorent le manque de ressources à ce niveau ainsi que la discrimination dont sont encore victimes les ex-détenus en dépit d'une certaine évolution des mentalités depuis quelques années.

Relativement au problème de l'alcoolisme présent chez certains libérés, les agents ne croient pas en la valeur de traitements forcés. Leurs interventions seront donc de nature à créer chez le client des motivations en vue de lui faire accepter une cure de désintoxication. L'agent peut également recourir à la suspension de la libération devant certaines rechutes mais cette mesure peut être évitée selon la patience de l'agent, sa compréhension du problème et sa volonté de supporter le client. Voici l'exemple de l'approche d'un agent face aux alcooliques :

Je comprends maintenant qu'un alcoolique va fonctionner pendant huit mois et puis que là, il va tout saboter : il va lâcher sa job, il va prendre une brosse, il va battre sa femme, tout ça [...]. Avant ça, je ne voulais rien savoir, je le retournais en-dedans, j'étais fầché. C'est plus çà maintenant parce que maintenant, je l'amène à se faire traiter. C'est que maintenant, je dis : tu as chuté, on recommence. Je travaille avec lui par exemple. Je travaille comme lui parce que c'est toujours cyclique un alcoolique; çà va toujours être de même, il faut que tu le prennes comme ça. Ca peut prendre des années avant qu'il en arrive à ne plus boire [...].

Toutefois, il demeure que les agents ne connaissent pas de solution-miracle à ce problème et, devant certains alcooliques chroniques, plusieurs agents se déclarent bien impuissants et désemparés. 
Au niveau conjugal, les libérés sont peu enclins à parler de leurs difficultés et les agents ne s'immiscent pas spontanément dans ce domaine de leur vie, préférant attendre que les clients abordent d'eux-mêmes ce sujet, ce qui arrive assez rarement. Puisque la mésentente conjugale est considérée comme une situation problématique susceptible de conduire à la récidive, les libérés craignent une suspension si l'agent découvre l'existence de difficultés de cet ordre.

Devant ces problèmes qui leur sont parfois rapportés par leurs clients ou par les épouses de ces derniers, l'ensemble des agents se sentent désarmés. Finalement, ils recommandent le recours à un conseiller matrimonial. Il est très rare qu'un agent s'implique davantage car il ne se sent pas qualifié pour intervenir au niveau de problèmes profonds. Voici l'opinion d'un agent à ce sujet :

[...] s'ils ont des problèmes au niveau sexuel ou des problèmes d'entente profonde, je ne me sens pas équipé pour travailler avec ça. J'aurais peur de toucher à des choses dont je ne serais pas capable de récupérer par après.

La recherche de solutions aux différents problèmes énoncés précédemment s'inscrit à l'intérieur d'une démarche orientée vers une aide que l'agent tente d'apporter aux libérés. Ce support apporté par l'agent s'allie à l'objectif premier de sa fonction qui est d'empêcher la récidive. Ainsi, dans son entreprise de resocialisation et d'amélioration de la personnalité de son client, l'agent tentera de mettre en valeur les qualités de celui-ci, l'aidera à acquérir un certain réalisme et à développer son sens de l'autonomie. De plus, l'agent essaiera de diriger le libéré vers de nouvelles activités ou de nouveaux milieux et il l'encouragera dans ses projets.

Malgré les efforts de l'agent, celui-ci rencontre de nombreux obstacles devant lesquels il se sent impuissant. Par exemple, les jeunes libérés non favorisés par les circonstances seraient plus prédisposés à la récidive. Dans ces cas, les interventions de l'agent viseront au moins à ce que leur récidive soit «moins forte, moins grave, moins violente». Et devant ces clients démunis, un agent se résoud à dire :

Comment tu veux demander à un gars de ne pas récidiver quand il n'a pas d'argent. Le pain, la commande coûtent la même chose pour tout le monde [...] tu as une survie élémentaire qu'il faut que tu assumes. C'est bien beau de 
phraser puis de dire : les gars ne veulent pas s'aider [...]. Mais quand même, il reste que c'est pas vrai tout le temps. Ils ont de la difficulté à se trouver des emplois et il n'y a rien qui est fait pour [...].

À l'égard des clients ayant de sérieux problèmes psychiatriques ou psychologiques, l'action de l'agent se limite à motiver le libéré en vue de lui faire accepter un traitement volontaire auprès de cliniciens spécialisés. Sur ce, un agent déclare : «(..) si on peut les introduire, on a fait notre devoir nous autres».

Bref, l'un de nos répondants résume ainsi les interventions des agents :

Moi, je trouve que notre action est très très terre-à-terre; c'est l'organisation matérielle de sa vie, l'organisation de ses loisirs, de ses activités, de ses centres d'intérêts. Je ne vais pas creuser dans son psychologique profond parce que je ne suis pas capable de faire ça et puis ce n'est pas tous les cas qui en ont besoin à part ça.

Empêcher la récidive demeure un objectif fondamental de la fonction de l'agent. Bien que les agents identifient nombre d'indices de récidive (instabilité d'emploi, problèmes financiers, difficultés conjugales, fréquentations douteuses, etc.), la dynamique de la récidive leur apparaît néanmoins comme une question énigmatique, un phénomène incompréhensible. Ainsi, décelant ou soupçonnant la présence d'indices chez un client, comment l'agent peut-il entreprendre une action efficace et cela, d'autant plus que même présents, ces indices ne conduisent pas irrémédiablement à la récidive? De plus, l'étonnement et l'impuissance des agents se manifestent lorsque survient une récidive que rien apparemment ne laissait présager.

Conscients des limites de leurs interventions, les agents ne peuvent qu'espérer apporter une certaine aide à leurs clients. Constamment dans l'incertitude quant à leur apport véritable, les agents situent l'essentiel de la relation d'aide au niveau d'une attitude attentive, compréhensive et intéressée envers leurs clients. Enfin, les agents n'escomptent atteindre que dans une proportion fort limitée une resocialisation adéquate résultant de leur action. Finalement, selon ceux-ci, le libéré demeure le premier responsable de sa réadaptation sociale.

Pour leur part, les libérés s'attribuent le mérite d'une réadaptation qui s'est faite graduellement. L'un d'eux mentionne : 
C'est beaucoup plus moi-même, le fait de mon attitude en général, mon évolution, là où j'étais rendu dans la vie.

(Parlant de son agent qui a une formation de psychologue)

[...] Ben comment est-ce qu'il voudrait m'aider, je ne suis pas intéressé à avoir son aide [...]. Quand je vois ou quand je pense à mon agent de libération conditionnelle, je vois ou je pense à un agent de libération conditionnelle; je ne le vois jamais ou je ne pense jamais à lui en tant que psychologue. D'ailleurs, si un jour j'avais besoin d'une aide psychologique quelconque, il ne serait même pas la dernière personne à qui je songerais en faisant mon choix. Et s'il était dentiste, je n'irais pas non plus me faire enlever une dent par lui.

L'opinion qui précède est une des réponses que pourrait recevoir certains agents qui se déclarent en mesure d'aider les libérés tout en constatant qu'ils ne peuvent intervenir du fait que leurs clients ne perçoivent pas leur compétence.

DILEMME AIDE ET CONTRÔLE

Notre étude soulève le dilemme aide-contrôle. En regard des deux aspects de leur fonction, nombre de situations obligent l'agent à faire un choix entre le contrôle pour lequel il est mandaté et l'aide qu'il croit plus susceptible d'apporter des résultats positifs à plus ou moins long terme.

II arrive en effet que, pour accentuer la relation d'aide, l'agent recommande au client qui a dérogé à une interdiction formelle de consommer des boissons alcooliques par exemple, de taire de telles dérogations sans quoi il se verra contraint d'appliquer des mesures cœrcitives. Suite aux aveux des clients relativement à certains bris de conditions ou à des offenses mineures, l'agent s'interroge sur le bien-fondé d'une éventuelle suspension. Sur ce, un agent définit sa position en ces termes :

De toute façon suspendre un gars de par les révélations qu'il fait, c'est pour moi, détruire toute possibilité de travail futur ou d'interventions futures de confiance avec ce gars-là.

À la lumière de nos données, il semble évident que les mesures d'aide et de contrôle se chevauchent constamment et que, selon l'interprétation qu'on leur donne, une même mesure peut être située dans l'un ou l'autre groupe d'interventions. Ainsi, le resserrement du rythme des rencontres tout comme la suspension " thérapeutique » sont perçues tantôt comme des mesures d'aide, tantôt comme des mesures de contrôle.

Cette ambivalence présente chez les agents n'existe pas chez les ex-détenus. Ces derniers distinguent nettement et prioritaire- 
ment l'aspect contrôle de la fonction d'aide au contraire des agents chez qui une ambiguïté subsiste. Pour les libérés, la menace constante d'un retour au pénitencier, l'éventualité d'une filature policière à la demande de l'agent, les intrusions de ce dernier dans sa vie ainsi que son questionnement toujours présent lors des rencontres ne s'interprètent que comme mesures de contrôle. Un des libérés soulève ainsi le double aspect de la fonction de l'agent :

[...]c'est faussé dès le départ. La meilleure façon de la caricaturer, c'est le gardien de prison avec une carabine sur l'épaule qui dit : «Ben je suis là pour t'aider ». Traverse la clôture pis il va te tirer dans la tête. Il va te dire : «Ben moi, il faut que je fasse ma job». Parfait! Il faut qu'il fasse sa job. Puis en même temps, il est sur l'autre bord et il essaie de se convaincre qu'il fait quelque chose d'utile ou d'humain.

L'agent, en contexte d'autorité où il est fortement identifié à un agent de répression, tente de distinguer son rôle de celui du policier. Pourtant, le double aspect de la fonction de l'agent revêt un caractère problématique comme l'indique l'un deux :

Vous voulez qu'on travaille au niveau de la réhabilitation sociale ou vous voulez qu'on travaille à la surveillance proprement dite? Là, c'est toujours là qu'ils nous disent : "Vous êtes là pour faire les deux 》. Alors, c'est où commence l'un, où arrête l'autre? C'est là la question.

CONCLUSIONS

La fonction de l'agent des libérations conditionnelles, telle que structurée par le Service correctionnel canadien, est axée sur le contrôle alors que l'agent poursuit prioritairement l'objectif d'aider les libérés : deux dimensions difficilement conciliables. Le libéré, ne percevant que l'aspect contrôle inhérent au rôle de l'agent, refuse l'aide que ce dernier souhaite lui apporter. Par ailleurs, il s'accommode assez bien des modalités de contrôle auxquelles il est soumis de par sa condition. Bref, selon notre étude, il apparaît que par rapport à la réinsertion sociale du libéré et à la prévention de la récidive, l'impact des interventions de l'agent est quasi inexistant.

D'autre part, les entrevues réalisées auprès des agents démontrent que ceux-ci ne croient généralement pas en la valeur des interventions imposées ou en l'efficacité des mesures répressives. De plus, les agents reconnaissent qu'une partie de leur clientèle n'a aucunement besoin de leur aide, ces libérés ne présentant pas de problèmes particuliers; leur réadaptation à la vie en société s'effectuera indépendamment de la présence de l'agent. Envers 
d'autres libérés pour qui une aide plus spécifique serait requise, les agents se sentent désemparés et impuissants à répondre adéquatement à leurs besoins.

À la lueur de nos résultats, tout nous porte à croire qu'une redéfinition du rôle de l'agent des libérations conditionnelles s'impose. Toutefois, une étude plus approfondie, tant au niveau de la cueillette des données que de leur analyse critique, nous permettrait de confirmer ou d'infirmer nos présentes conclusions. 